\title{
A Shopping-path Length Estimation Using Markov- chain-based Shopper Dynamics Model
}

\author{
Shunichi Ohmori \\ Department of Industrial and Management System Engineering, \\ Waseda University, Okubo 3-4-1, Shinjuku, Tokyo 169-8555 Japan \\ Email: ohmori0406@aoni.waseda.jp (Corresponding Author) \\ Masao Ueda \\ Department of Industrial and Management System Engineering, \\ Waseda University, Okubo 3-4-1, Shinjuku, Tokyo 169-8555 Japan \\ Email: m_ueda@aoni.waseda.jp \\ Kazuho Yoshimoto \\ Department of Industrial and Management System Engineering, \\ Waseda University, Okubo 3-4-1, Shinjuku, Tokyo 169-8555, Japan \\ Email: kazuho@waseda.jp
}

\begin{abstract}
We propose a framework of estimating shopping-path length, in which the floor is represented by the graph $G(V, E)$ with a vertex set $V$ and an arc set $E$ and the shopping-path length is measured by the number of zones (vertices) shoppers visit. We used the Markov-chain to model the dynamics of distribution of shoppers on the vertecies in the graph. We derive the (discrete) probability distribution of shopping path length using the transition matrix in the Markov-chain, and derive the expected path length. We proposed the index called the improvement importance index to quantify how local changes in the transition probability affect the entire shopping path length. We have tested our framework to the test data from an industrial application and the estimated path-length is compared to the actual one. We have a result that the error of estimation is $0.2 \%$.
\end{abstract}

Keywords: vehicle routing, logistic, supply chain management

\section{INTRODUCTION}

There is a strong consensus among both academia and industry that many purchases in a retail store are unplanned. For examples, Underhill 2009 claimed that "Supermarkets are places of high impulse buying..., 60 to 70 percent of purchases there were unplanned, grocery industry studies have shown us". These facts make shoppers' in-store behavior studies an important topic.

In order to increase the unplanned buying, it is important to know what factors that affect the number of purchases. One approach to analyze this is to divide a shopping path into a series of three exhaustive sequential and interrelated decisions the visit-shop-buy (Sorensen 2016, Hui et al. 2009). Using this double-conversion process we can divide the number of items purchased as follows:

$$
\boldsymbol{n}_{b}=\boldsymbol{n}_{v} \times \boldsymbol{p}_{s} \times \boldsymbol{p}_{b},
$$

where $n_{b}$ is the number of items bought (purchased), $n_{v}$ is the number of items visited (reached), $p_{s}$ is conversion rate from visitors to shoppers, and $p_{b}$ is the conversion rate from shoppers to buyers. This distinction is important, because it allows us to take different in-store marketing activities depending on which of conversions to fail. The first measure $n_{v}$ plays a critical role in the effectiveness of in-store marketing activities, while the other two measures $p_{s}$ and $p_{b}$ can be rather accounted for by products' attractiveness itself. To estimate $n_{v}$, it is necessary to understand shopping behaviours of customers deeply. shopping behaviours, however, is a very complex dynamical system. Thus, it is difficult to estimate how design factors (e.g., at-shelf promotions, aisle structure, product arrangement, or in-store communications) can influence dynamics of shopping trip.

In this study we propose a framework of estimating shopping-path length, in which the floor is represented by the graph $G(V, E)$ with a vertex set $V$ and an edge set $E$ and the shopping-path length is measured by the number of zones (vertices) shoppers visit. We used the Markov- chain to model the dynamics of distribution of shoppers on the vertecies in the graph. We derive the (discrete) probability distribution of shopping path length using the transition matrix in the Markov-chain and derive the expected path length. We proposed the index called the improvement importance index to quantify how local changes in the transition probability affect the entire shopping path length. We have tested our framework to the test data from an industrial application and the estimated path-length is compared to the actual one.

The reminder of this paper is as follows. In section 2 , we review the existing literatures. In section 3, we explain our proposed model. In section 4, we describe the field data used to estimate our model. We then discuss our results and managerial implications based on the behavioral findings. In section 5, we provide conclusion. 


\section{PROPOSED MODEL}

It is known from prior research that the shopping trips vary in length depending on various factors. Those factors include shopping mission (Walters and Jamil 2003), the day of the week (Kahn and Schmittlein 1989; Tanskanen et al. 2002), store type (Sorensen 2009) and store layout (Sorensen 2010) Several papers compare shopping lengths across countries (i.e. Sorensen 2010, Hui et al. 2009b). Sorensen 2016 studied data collected on 75,000 shoppers across a series of three stores to develop behavioral segmentation of shoppers. They developed three segmentations from mathematical clustering as quick, fill-in, stock-up. They discovered those three segments vary in shopping path length. Similarly, Inman, Winer, and Ferrari 2009 divide shoppers into three groups according to whether they visited 'all aisles', 'most aisles', 'a few aisles'.

Relationship Between In-Store Travel Distance and Unplanned Spending is also studied (Kollat and Willett 1967, Rock1987, Granbois 1968, Hui et al. 2009c). Kollat and Willett 1967 claimed that "in-store stimuli usually reminds shoppers of present or future needs rather than evoking new needs". Therefore, the longer the distance shoppers walk in the store, the more exposure to in-store stimuli to remind them of such potential needs. Other metrics to use are store-coverage (Granbois 1968, Hansen and Ottar Olsen 2006, Hui et al. 2013, Knox et al. 2011; Kollat and Willett 1967), the time spent in the store (Granbois 1968), and the number of aisles shopped (Granbois 1968), each of which has correlation with the unplanned buying.

Shopper marketing strategies aimed at increasing travel distance studied based on the observation shoppers' behaviors. Granbois 1968 and Iyer 1989 studied the product relocation strategy and discuss the effect of placing popular products in scattered locations throughout the store, forcing shoppers to cover a longer distance in the store. Hui et al., (2013) studied in-store mobile promotion strategies and revealed that strategically promoting three product categories through mobile promotion could increase unplanned spending by $16.1 \%$.

Recent research has examined many other factors that drive unplanned purchases. The factors include budget deviation (Stilley, Inman, and Wakefield 2010), browsing and shopping (Hui et al. 2009), social influences (Zhang et al. 2014), and factors that influence unplanned purchases and spending (Bell, Corsten, and Knox 2011, Hui et al. 2013, Inman, Winer, and Ferraro 2009). While this research stream has developed valuable managerial insights, most of the factors are very hard to control directly by retailers.

Our research makes two contributions to the literatures on the prior in-store studies. First, we build a model that can be estimated only from the traffic data. With recent advances in the RFID technologies, it is much easier to obtain the traffic data. Second, our model can quantify how local changes in the transition probability affect the entire shopping path length. Individual activities in the store are often interconnected, and thus, actions that attract shoppers in one zone can end up exacerbating another. With our model, it is easier to examine how local changes in design factors can influence dynamics of shopping trip and path length as a whole. This feature will help the retail manager compare alternative action plans in the context of what-if simulation.

\section{PROBLEM FORMULATION}

\subsection{Floor Representation}

Let $G(V, E)$ denotes a graph represented by $V$ and a set of arcs $E$ between nodes respectively.

- $G(V, E)$ : A graph represented by A set of nodes $V$ and a set of edges $E$

- $\quad V=\{0,1, \cdots, n, n+1\}$ : A set of nodes (0: Entrance, $1, \cdots, n$ :In-store zones, $n+1$ :Exit)

- $\pi_{t}=\left[\pi_{t 1}, \cdots, \pi_{t n}\right]^{T}$ : The number of customers who is visiting node $i$ at time $t$

- $\quad p_{i j}$ : Transition probability from node $i$ to node $j$ for $\forall(i, j) \in E$

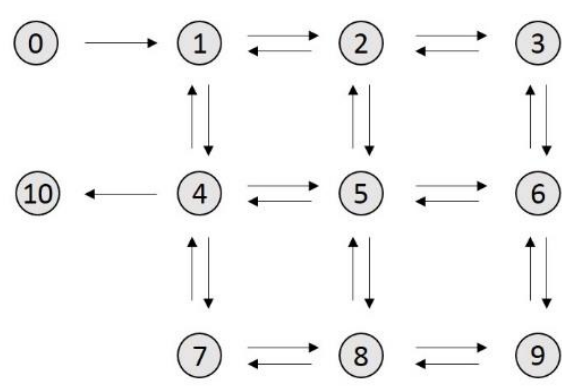

Figure 1 An example of graph of 11 nodes

\subsection{Spatial Distribution of Shoppers}

Let $\pi_{t i}$ is the probability of a shopper stays at node $i$ at period $t . \pi_{t}=\left[\pi_{t 1}, \cdots, \pi_{t n}\right]^{T}$ can be interpreted as the shopper's spatial distribution at node $i$ at period $t$. By the law of total probability, $\pi_{(t+1) j}$ can be derived as follows:

$$
\begin{aligned}
& \quad \operatorname{prob}\left\{x_{t+1}=j\right\}=\sum_{i} \operatorname{prob}\left\{x_{t+1}=j \mid x_{t}=i\right\} \operatorname{prob}\left\{x_{t}\right. \\
& \Leftrightarrow \pi_{(t+1) j}=\sum_{i} p_{i j} \pi_{t i} \\
& \Leftrightarrow \pi_{t+1}=P^{T} \pi_{t},
\end{aligned}
$$

where $P$ is the transition probability matrix with element $p_{i j}$.

From this equation, we have $\pi_{t}$ for $\forall t$ as

$$
\pi_{t}=\left(P^{t}\right)^{T} \pi_{0} \text {. }
$$

For steady state, we get $\pi=\left(P^{\infty}\right)^{T} \pi_{0}$.

We assume the Markov property of $p_{i j}$ as

$p_{i j}=\operatorname{prob}\left\{x_{t+1}=j \mid x_{t}=i_{t}, x_{t-1}=i_{t-1}, \cdots, x_{0}=i_{0}\right\}$

$=\operatorname{prob}\left\{x_{t+1}=j \mid x_{t}=i_{t}\right\}$

where $x_{t}$ is the node at which a shpper visits at period $t$. The Markov property is interpreted that the $x_{t+1}$ is determined only by the last step $x_{t}$. This assumption is not realistic if we consider the behavior of a particular shopper, because transition probability from node $i$ to node $j$ is clearly dependent on what the shopper has done. However, if we consider the behavior of a group of a large number of shoppers, this assumption becomes justified by the law of large numbers.

The example of transition probability of the graph described in Figure $\mathbf{2}$ is given as follows: 


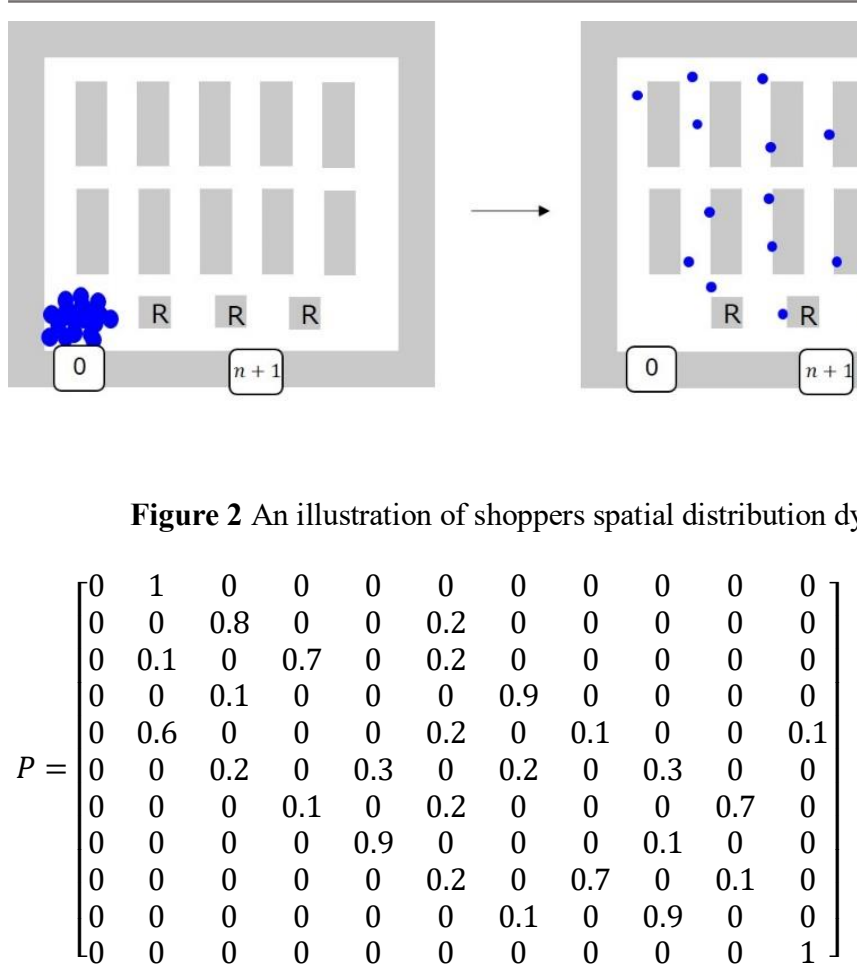

We see $p_{01}=1$ that the entrace node 0 is the source node and $p_{(10)(10)}=1$ that exit node 10 is the sink node. For example, with $\pi_{0}=[1,0,0,0,0,0,0,0,0,0,0]^{T}$ that the all shoppers stay at the entrance node 0 , dynamics of shoppers' distribution is described as follows:

$\pi_{t}=\left[\begin{array}{cccccccccccccc}t & 0 & 1 & 2 & 3 & 4 & 5 & \cdots & 10 & \cdots & 20 & \cdots & 50 & \cdots \\ \pi_{t 0} & 1 & 0 & 0 & 0 & 0 & 0 & \cdots & 0 & \cdots & 0 & \cdots & 0 & \cdots \\ \pi_{t 1} & 0 & 1 & 0 & 0.1 & 0 & 0.03 & \cdots & 0 & \cdots & 0 & \cdots & 0 & \cdots \\ \pi_{t 2} & 0 & 0 & 0.8 & 0 & 0.18 & 0 & \cdots & 0.07 & \cdots & 0.02 & \cdots & 0 & \cdots \\ \pi_{t 3} & 0 & 0 & 0 & 0.56 & 0 & 0.18 & \cdots & 0 & \cdots & 0 & \cdots & 0 & \cdots \\ \pi_{t 4} & 0 & 0 & 0.2 & 0 & 0.10 & 0 & \cdots & 0.25 & \cdots & 0.06 & \cdots & 0 & \cdots \\ \pi_{t 5} & 0 & 0 & 0 & 0.2 & 0 & 0.18 & \cdots & 0 & \cdots & 0 & \cdots & 0 & \cdots \\ \pi_{t 6} & 0 & 0 & 0 & 0 & 0.54 & 0 & \cdots & 0.09 & \cdots & 0.03 & \cdots & 0 & \cdots \\ \pi_{t 7} & 0 & 0 & 0 & 0.02 & 0 & 0.05 & \cdots & 0 & \cdots & 0 & \cdots & 0 & \cdots \\ \pi_{t 8} & 0 & 0 & 0 & 0 & 0.06 & 0 & \cdots & 0.17 & \cdots & 0.04 & \cdots & 0 & \cdots \\ \pi_{t 9} & 0 & 0 & 0 & 0 & 0 & 0.39 & \cdots & 0 & \cdots & 0 & \cdots & 0 & \cdots \\ \pi_{t 10} & 0 & 0 & 0 & 0.12 & 0.12 & 0.18 & \cdots & 0.43 & \cdots & 0.85 & \cdots & 1 & \cdots\end{array}\right]$

We see that at $t=10,43 \%$ of shoppers will get into the exit node, and at $t=50$, all shoppers get into the exit node. Figure 2 illustrates how this spatial distribution is changed over time.

\subsection{Parameter Estimation}

Let $Y_{k}=\{0,1\}$ be the random variable to take one if $k$ th shopper stayed at node $i$ moves to node $j$ and take zero otherwise, and $y_{k}$ be the realization of $Y_{k}$. Therefore, this process can be interpreted as the Bernoulli process $\left[Y_{1}, \cdots, Y_{N_{i}}\right]^{T}=\left[y_{1}, \cdots, y_{N_{i}}\right]^{T}$ with $N_{i}$ times trial.

Let $N_{i}$ be the total number of visits at node $i$, and $N_{i j}$ be the total number of shoppers to move from node $i$ to node $j$. The maximum likelihood of Bernouli process is as follows:

$$
\begin{aligned}
L\left(y_{1}, \cdots, y_{N_{i}} \mid p_{i j}\right) & =\Pi_{k=1}^{N_{i}} p_{i j}^{y_{k}}\left(1-p_{i j}\right)^{\left(1-y_{k}\right)} \\
& =p_{i j}^{\sum_{k=1}^{N_{j}} y_{k}}\left(1-p_{i j}\right)^{\sum_{k=1}^{N_{j}}\left(1-y_{k}\right)} \\
& =p_{i j}^{N_{i j}}\left(1-p_{i j}\right)^{\left(N-N_{i j}\right)} \\
\log L\left(y_{1}, \cdots, y_{N_{i}} \mid p_{i j}\right) & =N_{i j} \log \left(p_{i j}\right)+\left(N-N_{i j}\right) \log \left(1-p_{i j}\right)
\end{aligned}
$$

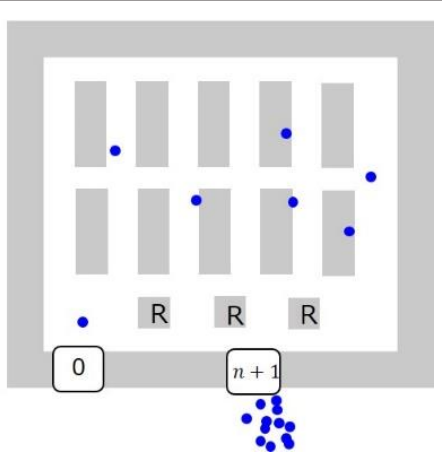

$$
\begin{aligned}
& \text { With } \frac{d \log L}{d p_{i j}}=0 \text {, we have } \\
& \quad \begin{aligned}
N_{i j} / p_{i j}-\left(N-N_{i j}\right) /\left(1-p_{i j}\right)=0 \\
\Leftrightarrow \quad p_{i j}=N_{i j} / N_{i}
\end{aligned}
\end{aligned}
$$

\subsection{Path Length Estimation}

In this section we describe our proposed method to estimate shopping path length. Let $p_{i j}^{m}$ be the conditional transition probability that a shopper stayed at node $i$ will stay at node $j$ after $m$ steps. The $p_{i j}^{m}$ is calculated as follows:

$$
p_{i j}^{m}=\left(P^{m}\right)_{i j}
$$

Using this expression, we can define $p_{0(n+1)}^{m}$ as transition probability from the entarance node 0 to the exit node $n+1$ after $m$ steps. For example, $P^{10}$ is calculated as follows:

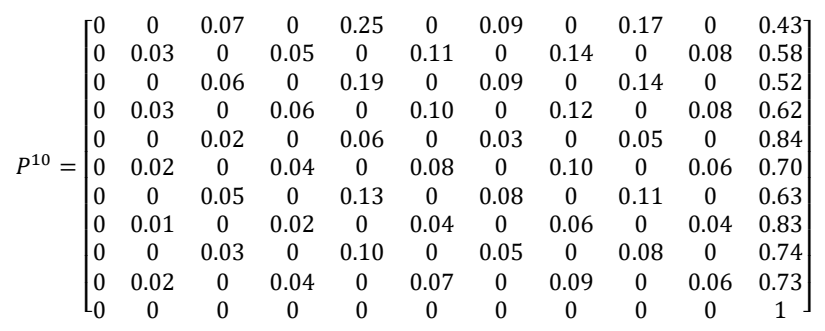

We see that the shopper stayed at the node 0 is distributed after 10 steps as $[0,0,0.07,0,0.25,0,0.09,0,0.17,0,0.43]^{T}$. For example, $43 \%$ of shopper stayed at node 0 will go to the exit node. Using this expression, we can form the cumulative probability distribution (CDF) of $n_{v}$ is as follows:

$$
F(m)=\operatorname{prob}\left(n_{v} \leq m\right)=p_{0(n+1)}^{m}
$$

Also, the probability distribution of $n_{v}$ can be calculated as follows:

$$
f(m)=\operatorname{prob}\left(n_{v}=m\right)=F(m)-F(m-1)
$$

Therefore, expected value of $n_{v}$ is calculated as:

$$
E\left(n_{v}\right)=\sum_{m=1}^{\infty} m \times f(m)
$$

As there is no closed form of $f(m)$, we calculate this length numerically. Practically, $f(m) \rightarrow 0$ quickly as $m \rightarrow$ $\infty$, so it is enough to have large number $N$ and calculate as

$$
E\left(n_{v}\right) \simeq \sum_{m=1}^{N} m \times f(m)
$$




\subsection{Improvement Importance Index}

To analyze the effect of improvement of marketing actions, e.g. in-store promotions, we propose the Improvement Importance Index. This is the index to quantify how the local changes in $p_{i j}$ will have an effect to an overall shopping-path length.

Let we assume that the transition probability from node $i$ to node $j$ is increased by some marketing actions, say, $p^{\prime}{ }_{i j}$ $:=p_{i j}+\Delta p_{i j}$. With this change, the transition probability from node $i$ to the other adjecent nodes is decreased. Let $N_{i}$ denote the set of adjacent nodes of node $i$, and $\widetilde{N}_{i j}=N_{i} \backslash$ is the set of adjecent nodes of node $i$ excluding the node $j$. We set the transition probability from node $i$ to $\forall k \in \widetilde{N}_{i j}$ by

$$
p_{i k}^{\prime}:=p_{i k} \times \frac{1-\Delta p_{i j}}{\sum_{l \in \widetilde{N}_{i j}} p_{i l}}, \forall k \in \widetilde{N}_{i j}
$$

We define $P^{\prime}$ is the transition probability matrix after improvement actions. We define the operatior $\frac{\partial}{\partial p_{i j}} E\left(n_{v}\right)$ as

$$
\frac{\partial}{\partial p_{i j}} E\left(n_{v}\right)=\lim _{\Delta p_{i j} \rightarrow 0} \frac{E\left(n_{v} \mid P^{\prime}\right)-E\left(n_{v} \mid P\right)}{\Delta p_{i j}},
$$

where $E\left(n_{v} \mid P\right)$ is the conditional expected number of $n_{v}$ given transition probability $P$. Again, as we do not have a closed expression, we calculate this value numerically. We call this value as Improvement Importance Index. By calculating this value, we can find which of arcs in the graph should be improved.

\section{CASE STUDY}

\subsection{Data Set}

The data set used in this case study is the customer survey data of shopping path in the grocery store located at Tokyo in Japan. In this survey, investigators chase shoppers and record walking path of each shopper.

- Time: AM10:00-PM9:00

- Day of the week: four successive days from Thursday to Sunday

- Sample size: 556 (see Table 1 for details)

The floor layout is presented as in Figure 3. The aisle space between gondolas and their intersections are divided into 170 zones and labeled as nodes. Adjacent nodes are connected by arcs. There are 170 nodes and 471 arcs, and the entrance node is located at left-upper corner and the registers are located at nodes 50, 52, 54, 56, 58, 60, 62, 64, 66 along the left edge, each of which is connected to the exit node 171 .

Table 1 Sample size for Age/Sex segments

\begin{tabular}{|c|c|c|}
\hline Age & Male & Female \\
\hline $20-30$ & 7 & 11 \\
\hline $30-40$ & 17 & 71 \\
\hline $40-50$ & 24 & 82 \\
\hline $50-60$ & 25 & 83 \\
\hline $60-70$ & 43 & 94 \\
\hline$\geq 70$ & 43 & 56 \\
\hline Total & 159 & 397 \\
\hline
\end{tabular}

Table 2 Top 10 Arcs of Improvement Importance Indices

\begin{tabular}{|c|c|c|}
\hline $\mathbf{i}$ & $\mathbf{j}$ & $\frac{\mathbf{\partial}}{\mathbf{\partial} \mathbf{p}_{\mathbf{i j}}} \mathbf{E}\left(\mathbf{n}_{\mathbf{v}}\right)$ \\
\hline 37 & 36 & 1.55 \\
\hline 37 & 161 & 1.54 \\
\hline 37 & 38 & 1.49 \\
\hline 165 & 163 & 1.39 \\
\hline 35 & 146 & 1.39 \\
\hline 35 & 34 & 1.39 \\
\hline 39 & 38 & 1.38 \\
\hline 165 & 164 & 1.36 \\
\hline 39 & 162 & 1.36 \\
\hline 165 & 169 & 1.34 \\
\hline 35 & 36 & 1.33 \\
\hline
\end{tabular}

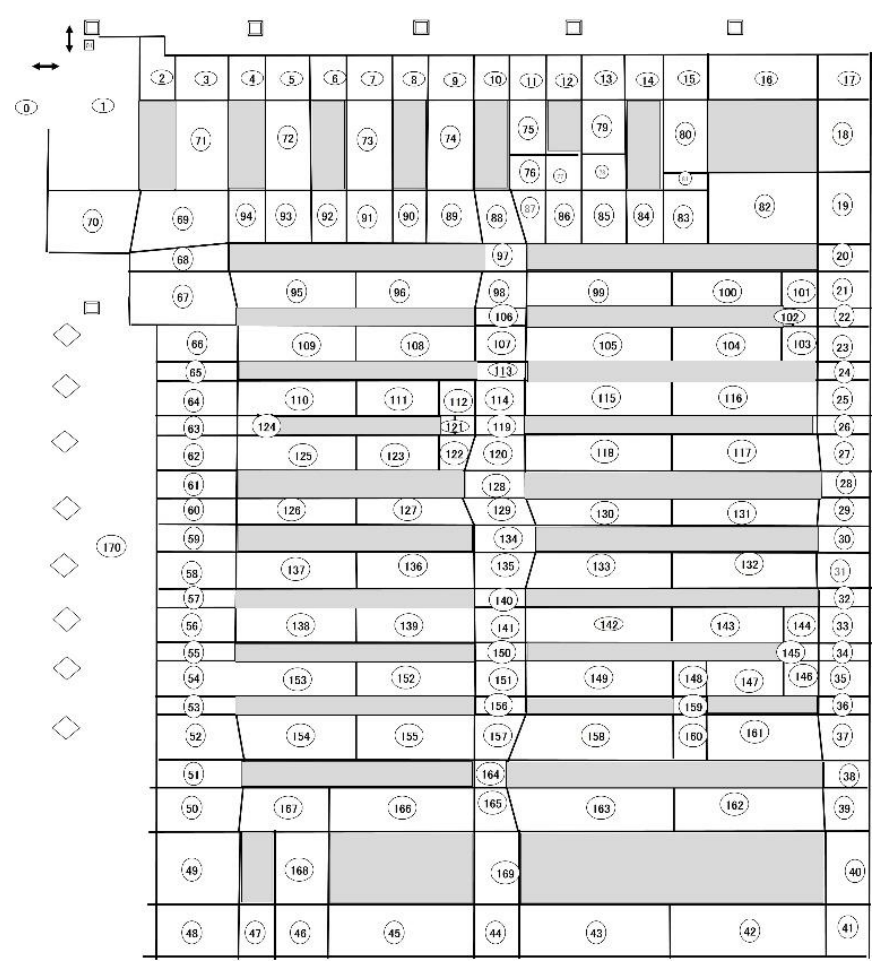

Figure 3 Floor layout

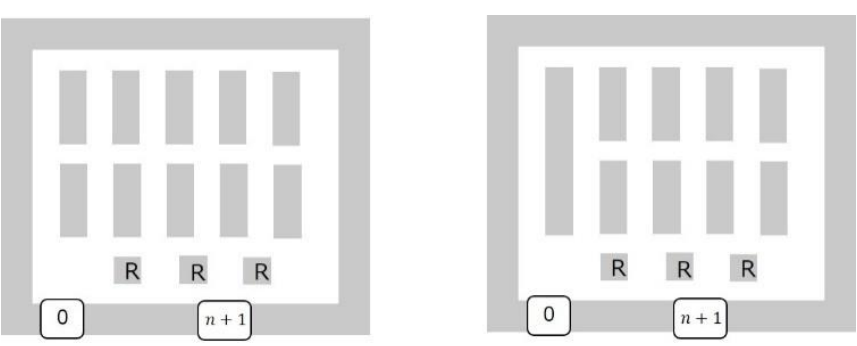

Figure 4 Aisle configurations (Left: Current Configuration, Right: Alternative Configuration)

\subsection{Analysis}

The result of estimation of path-length $E\left(n_{v}\right)$ and average path-length $\bar{n}_{v}$ of 556 shoppers are given as follows:

- $E\left(n_{v}\right)=91.6$

- $\bar{n}_{v}=91.4$

Estimation error is only $0.2 \%$, and thus, we see that our method could guess the path-length properly. The improvement importance indices for 471 arcs are calculated. Of all 471 arcs, the top 10 arcs are given as in Table 2. All of nodes in top 10 arcs are located right-bottom corner of the 
floor. This suggests that if the retailer have a marketing actions around right-bottom corner, shoppers tend to stay longer. This will help the retailer focus on their effort.

We consider the effect of aisle configuration. From the analysis of the improvement importance index, we consider an alternative configuration as presented in Figure 4. In the alternative configuration, we eliminate one middle cross aisle between the two most left aisles. By doing so, we expect that much more shoppers are forced to move to the rightbottom corner, where there are many arcs with higher improvement importance index.

For the calculation of the expected path-length, we set $p_{(88)(97)}=p_{(88)(97)}=p_{(97)(98)}=p_{(98)(97)}=0$ where the arcs $(88,97)$ and $(97,98)$ are in the cross-aisle. We increase the transition probability for the $\operatorname{arc} \forall k \in N_{(88)(97)}$ and $\forall k \in$ $N_{(97)(98)}$ as the same approach in the section 3.5 as $p_{i k}:=$ $\frac{p_{i k}+p_{i j}}{\left|N_{i j}\right|}$. The result of consideration is given as follows:

- Current Configuration: 91.6

- $\quad$ Alternative Configurations: 95.1

Therefore, we see that eliminating cross-aisle will force shoppers to move along outer track towards right-bottom corner.

\section{CONCLUSIONS REMARKS}

In this study, we propose a framework of estimating shopping-path length. We model the dynamics of spatial distribution of shoppers in the floor using Markov-chain. We derive the (discrete) probability distribution of shopping path length using the transition matrix in the Markov-chain and derive the expected path length. We proposed the index called the improvement importance index to quantify how local changes in the transition probability affect the entire shopping path length. We have tested our framework to the test data from an industrial application and the estimated path-length is compared to the actual one. We have a result that the error of estimation is $0.2 \%$. We compare two aisle configurations and estimate the benefit of eliminating a cross-aisle from the current configuration.

For future research, we are interested in testing our model to different data sets to validate the model. Especially, we are interested in comparing path-length of different size of stores. We also consider more comprehensive discussion about the relationship between design factors (e.g., at-shelf promotions, aisle structure, product arrangement, or in-store communications) and its potential benefits using our model.

\section{ACKNOWLEDGEMENT}

We wish to thank the Distribution Economics Institute of Japan for providing the data set used in this paper.

\section{REFERENCES}

Bell, D. R., Corsten, D., \& Knox, G. (2011). From point of purchase to path to purchase: How preshopping factors drive unplanned buying. Journal of Marketing, 75(1), pp. 31-45.

Granbois, D. H. (1968). Improving the study of customer instore behavior. Journal of Marketing, 32 (4) pp. 28-33.
Hansen, K., \& Olsen, S. O. (2006). Impulsive Buying Behavior-The Role of Impulse Buying Tendencies and Convenience Orientation. In ANZMAC 2007 Conference, University of Otago, New Zealand, December 3 (Vol. 5).

Hui, S. K., Inman, J. J., Huang, Y., \& Suher, J. (2013). The effect of in-store travel distance on unplanned spending: Applications to mobile promotion strategies. Journal of Marketing, 77(2), pp. 1-16.

Hui, S. K., Bradlow, E. T., \& Fader, P. S. (2009). Testing behavioral hypotheses using an integrated model of grocery store shopping path and purchase behavior. Journal of Consumer Research, 36(3), pp. 478-493.

Hui, S. K., Fader, P. S., \& Bradlow, E. T. (2009). Research note-the traveling salesman goes shopping: The systematic deviations of grocery paths from TSP optimality. Marketing Science, 28(3), pp. 566-572.

Hui, S. K., Fader, P. S., \& Bradlow, E. T. (2009). Path data in marketing: An integrative framework and prospectus for model building. Marketing Science, 28(2), pp. 320335 .

Inman, J. J., Winer, R. S., \& Ferraro, R. (2009). The interplay among category characteristics, customer characteristics, and customer activities on in-store decision making. Journal of Marketing, 73(5), pp. 1929.

Kahn, B. E., \& Schmittlein, D. C. (1989). Shopping trip behavior: An empirical investigation. Marketing Letters, 1(1), pp. 55-69.

Knox, G., Bell, D. R., \& Corsten, D. (2011). Situational determinants of unplanned buying in emerging and developed markets. Marketing Science Institute Working Paper Series. Marketing Science Institute.

Kollat, D. T., \& Willett, R. P. (1967). Customer impulse purchasing behavior. Journal of Marketing Research 4(1), pp. 21-31.

Rook, D. W. (1987). The buying impulse. Journal of Consumer Research, 14(2), pp. 189-199.

Sorensen, H., 2010. Tell 'em Where to Go; Tell 'em Which to Buy!, Views. TNS Global, Online.

Sorensen, H. (2016). Inside the mind of the shopper: The science of retailing. FT Press.

Stilley, K. M., Inman, J. J., \& Wakefield, K. L. (2010). Spending on the fly: mental budgets, promotions, and spending behavior. Journal of Marketing, 74(3), pp. 3447.

Tanskanen, K., Yrjola, H., \& Holmstrom, J. (2002). The way to profitable Internet grocery retailing-six lessons learned. International Journal of Retail and Distribution Management, 30(4), pp. 169-178.

Underhill, P. (2009). Why we buy: The science of shoppingupdated and revised for the Internet, the global consumer, and beyond. Simon and Schuster.

Walters, R. G., \& Jamil, M. (2003). Exploring the relationships between shopping trip type, purchases of products on promotion, and shopping basket profit. Journal of Business Research, 56(1), pp. 17-29.

Zhang, X., Li, S., Burke, R. R., \& Leykin, A. (2014). An examination of social influence on shopper behavior using video tracking data. Journal of Marketing, 78(5), pp. 24-41. 
Shunichi Ohmori (PhD) is an assistant professor at department of industrial \& system engineering, Waseda University in Japan, and a researcher at institute of global production \& logistics at Waseda University, and a researcher at data science institute at Waseda University. He received the master and Ph.D degree in engineering at Waseda University. His research interest lies in operations research and supply chain management.

Masao Ueda (PhD) is a professor at department of business design management, Waseda University in Japan, and a researcher at data science institute at Waseda University. He received the master degree in agriculture at Hokkaido University and Ph.D degree in commerce at Waseda University. His research interest lies in marketing engineering and marketing research.

Kazuho Yoshimoto (Dr.Engg) is a professor at department of industrial \& system engineering at Waseda University in Japan, and a head of Institute of Global Production \& Logistics at Waseda University. He received the master and Ph.D degree in engineering at Waseda University. His research interest lies in facility and logistics design. 